\title{
A role for the locus coeruleus in the modulation of feeding
}

Natale R. Sciolino ${ }^{1}$, Christopher M. Mazzone ${ }^{1}$, Nicholas W. Plummer ${ }^{1}$, Irina Evsyukova ${ }^{1}$, Jaisal Amin ${ }^{1}$, Kathleen G. Smith ${ }^{1}$, Christopher A. McGee ${ }^{2}$, Sydney A. Fry ${ }^{1}$, Cindy X. Yang ${ }^{1}$, Jeanne M. Powell $^{1}$, Michael R. Bruchas ${ }^{3}$, Alexxai V. Kravitz ${ }^{4}$, Jesse D. Cushman ${ }^{1}$, Michael J. Krashes ${ }^{5}$, Guohong Cui ${ }^{1}$, Patricia Jensen ${ }^{1 *}$.

${ }^{1}$ Neurobiology Laboratory, National Institute of Environmental Health Sciences, National Institutes of Health, Dept. of Health and Human Services, Research Triangle Park, NC, USA.

${ }^{2}$ Comparative Medicine, National Institute of Environmental Health Sciences, National Institutes of Health, Department of Health and Human Services, Research Triangle Park, NC, USA.

${ }^{3}$ Departments of Anesthesiology and Pharmacology, Center for the Neurobiology of Addiction, Pain, and Emotion, University of Washington, Seattle, WA, USA.

${ }^{4}$ Department of Psychiatry, Washington University, St Louis, MI, USA.

${ }^{5}$ National Institute of Diabetes and Digestive and Kidney Diseases, National Institutes of Health, Dept. of Health and Human Services, Bethesda, MD, USA.

Correspondence to:

${ }^{*}$ Patricia Jensen, Ph.D.

Neurobiology Laboratory

National Institute of Environmental Health Sciences

111 T.W. Alexander Drive

Bldg. 101, Rm. F118 (Mail Drop F1-11)

Research Triangle Park, NC 27709

Tel.: 984-287-3413

email: patricia.jensen@nih.gov 
SUMMARY: Noradrenergic neurons of the locus coeruleus (LC-NE) are known to play an important role in arousal, emotion, memory and cognition. In the present study, we use fiber photometry combined with chemogenetic and optogenetic approaches to demonstrate a previously unrecognized role for LC-NE neurons in the modulation of feeding. We show that endogenous activity of LC-NE neurons is enhanced while approaching food and suppressed during feeding. These changes in LC activity during feeding behavior are attenuated as mice approach satiety, demonstrating that nutritional state modulates LC responses to food. Direct activation of LC-NE neurons results in the suppression of feeding. Further, activation of an LC projection to the lateral hypothalamus also suppresses feeding. Together, these findings demonstrate a direct causal role for LC-NE activity in the modulation of feeding.

KEYWORDS: locus coeruleus, norepinephrine, feeding, metabolism, photometry, lateral hypothalamus, optogenetics, chemogenetics, anxiety, and aversion. 


\section{INTRODUCTION}

Evidence that noradrenergic neurons of the locus coeruleus (LC-NE) play a role in arousal was first established in a series of electrophysiology experiments conducted over four decades ago. These studies consistently showed that LC neurons in several different species are phasically activated during high-arousal behavioral states (e.g., orienting, waking, startle) and in response to salient sensory stimuli ${ }^{1-5}$ (reviewed in ${ }^{6,7-9}$ ). More recent studies using optoand chemogenetic approaches have demonstrated that LC-NE activity is both necessary and sufficient for promoting behavioral arousal, including sleep-to-wake transitions and emergence from anesthesia ${ }^{10,11}$.

In contrast, much less is known about the contribution of LC-NE activity to low-arousal behavioral states (e.g. sleeping, grooming, feeding). One early electrophysiology study noted that LC-NE activity is transiently decreased in rats during sleeping, grooming, and drinking a sucrose reward ${ }^{2}$. In a second study, decreased LC activity was observed in two monkeys eating apples $^{12}$. While these observations suggest that LC-NE activity may play a role in modulating feeding, this possibility has not previously been tested. In the present study, we used fiber photometry combined with chemogenetic and optogenetic approaches to test the hypothesis that activity of LC-NE neurons is involved in the modulation of feeding.

\section{RESULTS}

To identify the natural activity patterns of LC-NE neurons during feeding behavior, we used fiber photometry to monitor fluorescence of the calcium sensor GCaMP6f in LC-NE neurons. We selectively target these neurons by co-injecting Flp-dependent GCaMP6f and tdTomato AAVs into the LC of $D b h^{F / p o}$ mice (LC ${ }^{G C a M P / t d T}$ ) (Fig. 1a). To control for changes in fluorescence due to movement, GCaMP6f signal was normalized to tdTomato to yield a fluorescent ratio using a spectral unmixing approach ${ }^{13}$. To verify our recording conditions were sensitive to detect LC activity, we presented mice a visual flash known to activate LC 
neurons $^{2,14}$. We observed the expected increase in LC-NE activity in response to the visual stimulus (Supplementary Fig. 1), validating that we can reliably detect changes in LC-NE activity in the behaving mouse.

To determine if LC activity is altered during feeding-related behaviors, $\mathrm{LC}^{\mathrm{GCaMP} / \mathrm{tdT}}$ mice were fasted overnight and photometry recordings were collected during a 1-hr session while mice approached and consumed food pellets from the feeding experimentation device (FED) ${ }^{15}$ (Fig. 1a). To minimize the effects of stress and novelty during the experiments, mice were habituated for several days to eat from the FED while in the testing arena. To determine if nutritional status influences LC responses during approach and consumption, we compared LC activity early and late in the session as mice approached satiety. Assessment of approach revealed an increase in activity of LC-NE neurons during approach early in the session. This approach-related LC response was abolished later in the session when mice had consumed more food (Fig. 1b-e), suggesting that energy levels modulate LC activity during appetitive behavior.

Assessment of consummatory responses revealed that LC-NE activity was rapidly suppressed during feeding (Fig. 1b-d). This decrease in LC activity was observed during consumption of pellets throughout the session (Fig. 1d). To determine if the transition from hunger to satiety had a gradual impact on food-related LC activity across the trial, we next performed a linear regression analysis. We found the suppression of LC-NE activity during feeding was significantly attenuated as fasted mice ate more within the trial $\left(R^{2}=0.049\right)$ (Fig. 1e), suggesting energy levels gradually modulate LC activity during consumption. Importantly, there was no significant change in baseline LC-NE activity (Supplementary Fig. 2) or movement velocity (Supplementary Fig. 3) as mice consumed more pellets across the session. Taken together, these findings suggest nutritional state-related changes in LC-NE activity during feeding-related behavior was not driven by alterations in movement. 
To determine whether activation of LC-NE neurons would directly influence feeding behavior, we employed an intersectional chemogenetic approach in which the excitatory Gqcoupled receptor hM3Dq is selectively expressed in noradrenergic neurons of the LC complex ${ }^{16}$ $\left(\mathrm{LC}^{\mathrm{hM} 3 \mathrm{Dq}}\right)$ (Fig. 2a). To test whether our chemogenetic strategy activates LC-NE neurons in vivo, we performed fiber photometry recordings in $\mathrm{LC}^{\mathrm{hM} 3 \mathrm{Dq}}$ mice and $\mathrm{LC}^{\mathrm{GFP}}$ littermate controls following administration of clozapine $\mathrm{N}$-oxide (CNO) or vehicle. Recordings revealed a sustained increase in LC-NE activity following CNO treatment in LC ${ }^{\mathrm{hM} 3 \mathrm{Dq}}$ mice compared to controls (Fig. 2b). To compare the magnitude of LC ${ }^{\mathrm{hM} 3 \mathrm{Dq}}$ activation to a well-known stimulator of LC activity ${ }^{17-22}$ and behaviors associated with stress and threat ${ }^{23-25}$, we next administered the inhibitory Gi-coupled alpha-2 adrenergic receptor antagonist yohimbine (3 mg/kg i.p.). As expected, the "pharmacological stressor" yohimbine increased LC-NE activity relative to vehicle control (Fig. 2b), and this effect was comparable in magnitude to LC ${ }^{\mathrm{hM} 3 \mathrm{Dq}}$ activation. Importantly, no effect of CNO or vehicle was observed in LC GFP controls (Fig. 2b), suggesting the dynamics observed were unrelated to mouse movement. Together, the findings establish that our chemogenetic approach is effective in stimulating LC-NE activity in vivo.

To test if activation of LC-NE neurons would suppress feeding in hungry mice, LC ${ }^{\text {hM3Dq }}$ mice and littermate controls were overnight fasted and then treated with $\mathrm{CNO}$ or vehicle before placement in a novel arena containing standard chow (Fig. 2c). In CNO-treated LC ${ }^{\mathrm{hM} 3 \mathrm{Da}}$ mice, we found activation of LC-NE neurons suppressed feeding (Fig. 2c). Importantly, CNO had no effect on the behavior of littermate controls (Fig. 2c). These findings suggest that, despite the influence of hunger, activation of LC-NE neurons suppresses feeding in fasted mice.

To identify precisely how LC activation influences energy balance, we simultaneously measured feeding and metabolism of $\mathrm{LC}^{\mathrm{hM} 3 \mathrm{Da}}$ and littermate control mice given drinking water with CNO (30 or $100 \mu \mathrm{g} / \mathrm{mL}$ ) or plain water on alternate days (Fig. 2d). In CNO-treated LC ${ }^{\text {hM3Dq }}$ mice, activation of LC-NE neurons dose-dependently suppressed feeding by reducing the amount of meals consumed, without affecting meal size (Fig. 2d). Circadian analysis revealed 
suppression of feeding occurred during lights-off when LC ${ }^{\mathrm{hM} 3 \mathrm{Dq}}$ mice drank the most CNO (Supplementary Fig. 4). This suppression of feeding was not observed during lights-on (Supplementary Fig. 4), indicating that LC-mediated suppression of feeding was specific and reversible. Using indirect calorimetry to measure energy expenditure and respiratory exchange rate, we observed LC-NE activation had no effect on these metabolic endpoints (Fig. 2d). Together these changes resulted in weight loss in CNO-treated LC ${ }^{\text {hM3Dq }}$ mice (Fig. 2d). CNO had no effect on measures of feeding or metabolism in littermate controls (Fig. $2 \mathbf{d}$ and Supplementary Fig. 4). These findings collectively demonstrate that activation of LC-NE neurons suppresses feeding without altering metabolism. Given our finding that LC activation selectively altered energy intake, in subsequent experiments we focused our observations to measures of feeding.

Since it is well known that LC-NE neurons co-express several neuropeptides ${ }^{26-29}$, we next tested whether LC-mediated suppression of feeding depends on NE signaling. To selectively disrupt NE synthesis in LC neurons, we crossed our dopamine $\beta$-hydroxylase (Dbh) conditional knockout mice with $E n 1^{\text {cre }}$ (LC ${ }^{D b h}$ mutants; unpublished reagent, NWP and PJ) (Fig. 2e). To increase LC activity ${ }^{17-19}$ (Fig. 2b) and NE release ${ }^{20-22}$, we pretreated LC ${ }^{D b h}$ mutants and littermate controls with yohimbine (3 mg/kg i.p.) or vehicle and measured food intake. We found that baseline food intake was similar between $L^{D b h}$ mutants and controls treated with vehicle (Fig. 2f). Food intake was significantly reduced in controls treated with yohimbine, but in contrast, yohimbine had no effect on feeding in LC ${ }^{D b h}$ mutants (Fig. 2f). Because lack of LC-NE is the only difference between the mutants and controls, these findings suggest that LC neurons require norepinephrine for the observed suppression of feeding evoked by yohimbine. Measures of body weight revealed similar weight gain in adult $L C^{D b h}$ mutants and controls (Fig. 2e), consistent with the full Dbh mutant that show deficits in NE does not produce excessive weight gain $^{30,31}$. 
Prior studies have shown that NE has a strong inhibitory effect when applied directly in the lateral hypothalamus area $(\mathrm{LHA})^{32-34}$. To determine if this effect is mediated by the LC, we measured Fos immunoreactivity in $\mathrm{LC}^{\mathrm{hM} 3 \mathrm{Da}}$ mice following treatment of $\mathrm{CNO}$ or vehicle. Activation of LC-NE neurons resulted in a significant reduction in Fos expression in the LHA (Supplementary Fig. 5a). To determine if this response was specific to the LHA, we measured Fos expression in two additional feeding-related targets of the LC, the dorsal medial and ventromedial hypothalamic nuclei. We observed no change in Fos expression in either nucleus (Supplementary Fig. 5b).

To directly test whether enhanced activity in the LC-LHA circuit suppresses feeding, we injected AAVs expressing cre-dependent channelrhodopsin-2 (ChR2) ${ }^{35,36}$ or eYFP in the LC of $D b h^{\text {cre }}$ mice (Fig. 3a). Consistent with prior observations ${ }^{37-39}$, we observed LC derived axons in the LHA that were labeled by eYFP (Fig. 3b-c). ChR2 $2^{\text {LC-LHA }}$ and eYFP mice were next fasted overnight and food intake was measured in the presence or absence of photostimulation (Fig. 3d). Given that high-power (20-mW total power) and high-frequency ( $>5 \mathrm{~Hz}$ ) photostimulation of LC-NE soma has been shown to elicit reversible behavioral arrest ${ }^{10}$, we used a photostimulation protocol $(10-\mathrm{Hz}, 10-\mathrm{ms}$ pulses, $\sim 7-\mathrm{mW}$ total power) that has been shown to elicit high tonic rates of LC firing without behavioral arrests ${ }^{38}$. We revealed that ChR2 ${ }^{\text {LC-LHA }}$ mice had suppressed feeding during photostimulation compared to eYFP controls (Fig. 3e). Importantly, photostimulation had no significant effect in eYFP controls and none of the manipulations affected locomotor activity (Fig. 3e-g). Using this photostimulation protocol, we never observed a mouse that was immobilized in the recorded video or non-responsive to the experimenter directly after testing, confirming behavioral arrests were not observed in the present study. Furthermore, photostimulation of ChR2-expressing LHA terminals did not induce antidromic activity of LC-NE neurons as measured by Fos (Supplementary Fig. 6).

To determine if a shorter duration of stimulation would suppress feeding, we next used an epoch-based design wherein photostimulation was presented in alternating epochs or 
withheld for the entire experiment in fasted mice (Fig. 3h). When photostimulation was withheld, we observed a similar amount of feeding between ChR2 $2^{\text {LC-LHA }}$ and eYFP groups, wherein food intake was gradually decreased across the experiment as mice transitioned from hunger to satiety (Fig. 3i). Similar feeding was also observed between ChR2 ${ }^{\text {LC-LHA }}$ and eYFP during the initial photostimulation epoch (Fig. 3i), indicating stimulation of the LC-LHA pathway has no effect when hunger levels are at peak following a fast. In contrast, ChR2 ${ }^{\text {LC-LHA }}$ mice had suppressed feeding during the subsequent photostimulation epoch when hunger dampened compared to controls (Fig. 3i-j). Taken together, the findings demonstrate stimulation of the LCLHA pathway has a suppressive effect on feeding that may be modulated by hunger levels.

Since direct activation of LC-NE neurons enhances aversion and anxiety-like behaviors $^{16,38,40}$, we next tested if activation of the LC-LHA pathway would also enhanced these emotion-related responses. We therefore measured anxiety-like behavior of ChR2 ${ }^{\mathrm{LC}-\mathrm{LHA}}$ and eYFP mice in the open field test (OFT) and elevated plus maze (EPM) during photostimulation (Supplementary Fig. 7a-b). We observed ChR2 $2^{\text {LC-LHA }}$ mice spent significantly less time in the center of the OFT (Supplementary Fig. 7c) and tended to spend less time in the open arms of the EPM (Supplementary Fig. 7e), demonstrating that stimulating the LC-LHA pathway is anxiogenic. To assess if stimulation of the LC-LHA pathway has a negative or positive valence, we employed a real-time place preference test (RTPT) that triggers photostimulation upon entry into a designated side of the arena. We found ChR2 $2^{\text {LC-LHA }}$ mice spent less time in the stimulationpaired side compared to eYFP controls (Supplementary Fig. 7g), indicating an aversive behavioral response resulting from LC-LHA circuit activation. Assessment of locomotor activity revealed $\mathrm{ChR} 2^{\mathrm{LC}-\mathrm{LHA}}$ mice had reduced ambulation in the OFT, but no change in the EPM and RTPT compared to eYFP control (Supplementary Fig. $\mathbf{7 d}, \mathbf{f}, \mathbf{h}$ ), suggesting the LC-LHA circuit does not have an overall impact on locomotion. Together, the findings demonstrate that activation of the LC-LHA pathway suppresses feeding, potentially due to an enhancement of negative valence. 
To test if inhibition of the LC-LHA circuit enhances feeding, we injected AAVs expressing cre-dependent halorhodopsin (eNpHR3.0-eYFP) or eYFP in the LC of Dbh ${ }^{\text {cre }}$ mice (Supplementary Fig. 8a-b). We next measured food intake in free-feeding eNpHR ${ }^{\text {LC-LHA }}$ mice and controls during optical illumination. We observed similar food intake between eNpHR ${ }^{\text {LC-LHA }}$ mice and eYFP controls (Supplementary Fig. 8c). These findings, together with our prior optogenetic results, suggest that activation of the LC-LHA pathway suppresses feeding, but inhibition of the pathway does not promote feeding.

\section{DISCUSSION}

Collectively, we have demonstrated that endogenous activity of LC-NE neurons is suppressed during feeding in a manner modulated by nutritional state. We also found that activation of LC-NE neurons resulted in suppression of feeding through release of norepinephrine. Our experiments demonstrate that endogenous activity of LC-NE neurons is dynamically modulated during the consummatory sequence, with initial activation during food approach followed by a suppression of LC activity during consumption. This native pattern of LC-NE activity during consumption is unlikely attributed to changes in sleep, learning, stress or anxiety as our experimental mice were habituated to all aspects of the assay and they were tested during the portion of the circadian cycle when they are the most active. Instead, the approach-related LC response we observed likely reflects an appetitive response to food, in agreement with prior electrophysiological studies that show burst firing of LC neurons is associated with Pavlovian appetitive behavior in monkeys (e.g., lipping) ${ }^{41}$. Given LC neurons are well-known to modulate arousal $\left.\right|^{6,7,42,43}$, the decrease in LC activity we observed during feeding might reflect a decrease in arousal and/or disengagement with external sensory inputs to facilitate consummatory behavior.

Interestingly, we uncovered that suppression of LC-NE activity during consumption was gradually attenuated as mice approached satiety, indicating a previously unrecognized influence 
of nutritional status on endogenous LC responses. Attenuation of the consummatory-related LC response could serve to direct attentional arousal from internal-to-external environment when energy levels transition from hunger to satiety, although additional research is needed to test this possibility. We also observed a loss of the approach-related LC response as hunger levels diminished, which may contribute to the decrease in salience of food as energy balance is restored. Such potential interpretation is congruent with integrative theories of LC-NE neurons which describe that this modulatory system is designed to optimize behavioral performance to a changing environment (as reviewed in ${ }^{6,7,9}$ ). These nutritional-state-related changes in LC-NE activity during feeding are not likely involved in coordinating some motor response, as locomotor activity remained stable across the trial, even as mice approached satiety (Supplementary Fig. 3).

While there have been hints that LC-NE neurons could be involved in the suppression of feeding $2,12,30,44-46$, this possibility had not been directly tested. Experimentally determining a causal role for the LC-NE activity in feeding remained elusive using traditional lesion and genetic approaches. Physical lesions of the LC area had not produced a consistent change in feeding behavior ${ }^{46-49}$, probably due to unreliable and nonselective effects. Further, although genetic ablation of dopamine beta-hydroxylase $(D b h)$ in mice had been linked to an elevation in food intake during cold stress ${ }^{30}$, the contribution of the LC had remained unclear as the full $D b h$ knockout impacts all noradrenergic/adrenergic neurons in both the central and peripheral nervous systems. In the present study, we used chemogenetics to reveal a causal role for LCNE activity in the suppression of feeding. Further, using our LC ${ }^{D b h}$ mutants, we found that loss of LC-NE prevents the suppression of feeding evoked by yohimbine, which activated LC neurons (Fig. 2b). Optogenetic stimulation of the LC $\rightarrow$ LHA pathway suppressed feeding while also enhancing aversion and anxiety-like behavior. In contrast, optogenetic inhibition of the LC-LHA pathway did not lead to an enhancement of feeding in freely feeding mice tested in a familiar cage. Together, our findings suggest that enhanced activity in LC-NE neurons suppresses 
feeding but inhibition does not promote feeding. In context of the boarder LC literature, our findings suggest that LC-NE neurons are involved in the modulation of feeding by integrating both external cues (e.g., anxiogenic environmental cues) and internal drives (e.g., nutritional state).

Over four decades ago, a series of seminal studies established that direct delivery of NE agonists into the LHA suppressed feeding ${ }^{50-54}$. Using optogenetics, we have now revealed two previously unrecognized roles for a LC to LHA circuit: (1) the suppression of feeding and (2) enhancement of negative valence behavior (i.e., anxiety, aversion). This dual function is coherent with the well-established literature that shows LHA cell-types drive a variety of complex behaviors, including feeding, anxiety, and arousal ${ }^{55-59}$. An important future direction will be to determine whether the feeding and anxiety-related changes following LC-LHA pathway stimulation are mediated by separate or overlapping processes (e.g., target cell-types, receptor signaling mechanisms). Many cell-types may be involved, as prior electrophysiological studies have shown that NE has an inhibitory effect on the majority of LHA neurons ${ }^{32-34,60,61}$.

Given the widespread projections of LC-NE neurons throughout the brain ${ }^{37}$, it is likely that projections beyond hypothalamus are also involved in LC-NE modulation of feeding. It will be important to test this possibility using newly available tools to map, monitor and manipulate specific LC noradrenergic circuits. In sum, our findings expand upon established theories of LC function, which classically focus on arousal, emotion, memory and sleep $6,7,42,43,62$, to include the modulation of feeding.

\section{METHODS}

Methods and associated references are available in the Supplemental information.

\section{SUPPLEMENTAL INFORMATION}

Supplemental information can be found with this article. 


\section{ACKNOWLEDGEMENTS}

We thank Madeline Hsaing, Kushal Prasad, and Janiece Morgan for technical assistance in behavioral and photometry experiments, as well as Juhee Haam for technical assistance with the photometry setup. We thank Social \& Scientific Systems (Sandra McBride, Matthew Bridge, and Mike Easterling) for the custom code used to analyze photometry data (contract HHSN273201600011C with NIEHS). Valuable support was provided by the NIEHS Neurobehavioral, Fluorescence Microscopy and Imaging, and Viral Vector Cores. We thank the NIMH Drug Supply Program for providing the CNO. This research was supported by the Intramural Research Program of the NIH, NIEHS (ES102805 to PJ, ES103310 to GC) and NIDDK (DK075087 and DK075089 to MJK), and the Extramural Research Program of NIH (NIMH MH112355 to MRB).

\section{AUTHOR CONTRIBUTIONS}

NRS and PJ conceived, designed and supervised the project. Photometry experiments were performed by NRS and CMM under the guidance of GC and supervision of JDC. Feeding and metabolic experiments were performed by NRS, and analyzed by CAM, JA, JMP and NRS under the guidance of AVK and MJK. Optogenetic experiments were performed by NRS under the guidance of MRB, MJK, and JDC. NP created the $D b h^{c r e}$ and $D b h^{c K O}$ alleles and Flpdependent viral vectors. IE characterized the $D b h^{c K O}$ allele. Immunohistochemistry, in situ hybridization, and image acquisition was performed by NRS, KGS, JA, CYK and JP. Cell counts were performed by SAF and CXY. NRS and PJ wrote the manuscript with input from coauthors.

\section{DECLARATION OF INTERESTS}

The authors declare no competing financial interests. 


\section{REFERENCES}

1 Hobson, J. A., McCarley, R. W. \& Wyzinski, P. W. Sleep cycle oscillation: reciprocal discharge by two brainstem neuronal groups. Science 189, 55-58, doi:10.1126/science.1094539 (1975).

2 Aston-Jones, G. \& Bloom, F. E. Norepinephrine-containing locus coeruleus neurons in behaving rats exhibit pronounced responses to non-noxious environmental stimuli. $J$ Neurosci 1, 887-900 (1981).

3 Rasmussen, K., Morilak, D. A. \& Jacobs, B. L. Single unit activity of locus coeruleus neurons in the freely moving cat. I. During naturalistic behaviors and in response to simple and complex stimuli. Brain Res 371, 324-334 (1986).

4 Grant, S. J., Aston-Jones, G. \& Redmond, D. E., Jr. Responses of primate locus coeruleus neurons to simple and complex sensory stimuli. Brain Res Bull 21, 401-410 (1988).

5 Herve-Minvielle, A. \& Sara, S. J. Rapid habituation of auditory responses of locus coeruleus cells in anaesthetized and awake rats. Neuroreport 6, 1363-1368 (1995).

6 Sara, S. J. \& Bouret, S. Orienting and reorienting: the locus coeruleus mediates cognition through arousal. Neuron 76, 130-141, doi:10.1016/j.neuron.2012.09.011 (2012).

7 Aston-Jones, G. \& Cohen, J. D. An integrative theory of locus coeruleus-norepinephrine function: adaptive gain and optimal performance. Annu Rev Neurosci 28, 403-450, doi:10.1146/annurev.neuro.28.061604.135709 (2005).

8 Berridge, C. W., Schmeichel, B. E. \& Espana, R. A. Noradrenergic modulation of wakefulness/arousal. Sleep Med Rev 16, 187-197, doi:10.1016/j.smrv.2011.12.003 (2012).

9 Aston-Jones, G. \& Waterhouse, B. Locus coeruleus: From global projection system to adaptive regulation of behavior. Brain Res 1645, 75-78, doi:10.1016/j.brainres.2016.03.001 (2016).

10 Carter, M. E. et al. Tuning arousal with optogenetic modulation of locus coeruleus neurons. Nat Neurosci 13, 1526-1533, doi:10.1038/nn.2682 (2010).

11 Vazey, E. M. \& Aston-Jones, G. Designer receptor manipulations reveal a role of the locus coeruleus noradrenergic system in isoflurane general anesthesia. Proc Natl Acad Sci U S A 111, 3859-3864, doi:10.1073/pnas.1310025111 (2014).

12 Grant, S. J. \& Redmond, D. E., Jr. Neuronal activity of the locus ceruleus in awake Macaca arctoides. Exp Neurol 84, 701-708 (1984).

13 Meng, C. et al. Spectrally Resolved Fiber Photometry for Multi-component Analysis of Brain Circuits. Neuron 98, 707-717 e704, doi:10.1016/j.neuron.2018.04.012 (2018). 
14 Foote, S. L., Aston-Jones, G. \& Bloom, F. E. Impulse activity of locus coeruleus neurons in awake rats and monkeys is a function of sensory stimulation and arousal. Proc Natl Acad Sci U S A 77, 3033-3037 (1980).

15 Nguyen, K. P., O'Neal, T. J., Bolonduro, O. A., White, E. \& Kravitz, A. V. Feeding Experimentation Device (FED): A flexible open-source device for measuring feeding behavior. J Neurosci Methods 267, 108-114, doi:10.1016/j.jneumeth.2016.04.003 (2016).

16 Sciolino, N. R. et al. Recombinase-Dependent Mouse Lines for Chemogenetic Activation of Genetically Defined Cell Types. Cell Rep 15, 2563-2573, doi:10.1016/j.celrep.2016.05.034 (2016).

17 Goldstein, J. M., Knobloch, L. C. \& Malick, J. B. Electrophysiological demonstration of both alpha 2-agonist and antagonist properties of RX 781094. Eur J Pharmacol 91, 101105, doi:10.1016/0014-2999(83)90368-0 (1983).

18 Pitts, D. K. \& Marwah, J. Electrophysiological actions of cocaine on noradrenergic neurons in rat locus ceruleus. J Pharmacol Exp Ther 240, 345-351 (1987).

19 Osaka, T. \& Matsumura, H. Noradrenergic inputs to sleep-related neurons in the preoptic area from the locus coeruleus and the ventrolateral medulla in the rat. Neurosci Res 19, 39-50 (1994).

20 Abercrombie, E. D., Keller, R. W., Jr. \& Zigmond, M. J. Characterization of hippocampal norepinephrine release as measured by microdialysis perfusion: pharmacological and behavioral studies. Neuroscience 27, 897-904 (1988).

21 Duggan, S., Hong, M., Milne, B. \& Jhamandas, K. The role of excitatory amino acids in the expression of precipitated acute and chronic clonidine withdrawal: an in vivo voltammetric study in the rat locus coeruleus. Brain Res 665, 253-261, doi:10.1016/0006-8993(94)91345-5 (1994).

22 Park, J., Kile, B. M. \& Wightman, R. M. In vivo voltammetric monitoring of norepinephrine release in the rat ventral bed nucleus of the stria terminalis and anteroventral thalamic nucleus. Eur J Neurosci 30, 2121-2133, doi:10.1111/j.14609568.2009.07005.x (2009).

23 Holmes, A., Yang, R. J. \& Crawley, J. N. Evaluation of an anxiety-related phenotype in galanin overexpressing transgenic mice. J Mol Neurosci 18, 151-165, doi:10.1385/JMN:18:1-2:151 (2002).

24 Bhattacharya, S. K., Mitra, S. K. \& Acharya, S. B. Anxiogenic activity of isatin, a putative biological factor, in rodents. J Psychopharmacol 5, 202-206, doi:10.1177/026988119100500304 (1991).

25 Thiebot, M. H., Soubrie, P., Doare, L. \& Simon, P. Evidence against the involvement of a noradrenergic mechanism in the release by diazepam of novelty-induced hypophagia in rats. Eur J Pharmacol 100, 201-205 (1984). 
26 Charnay, Y. et al. Evidence for the presence of enkephalin in catecholaminergic neurones of cat locus coeruleus. Neurosci Lett 30, 147-151 (1982).

27 Sofroniew, M. V. Vasopressin- and neurophysin-immunoreactive neurons in the septal region, medial amygdala and locus coeruleus in colchicine-treated rats. Neuroscience 15, 347-358 (1985).

28 Cortes, R., Ceccatelli, S., Schalling, M. \& Hokfelt, T. Differential effects of intracerebroventricular colchicine administration on the expression of mRNAs for neuropeptides and neurotransmitter enzymes, with special emphasis on galanin: an in situ hybridization study. Synapse 6, 369-391, doi:10.1002/syn.890060410 (1990).

29 Nishio, T. et al. Cellular localization of nerve growth factor-like immunoreactivity in adult rat brain: quantitative and immunohistochemical study. Neuroscience 60, 67-84 (1994).

30 Thomas, S. A. \& Palmiter, R. D. Thermoregulatory and metabolic phenotypes of mice lacking noradrenaline and adrenaline. Nature 387, 94-97, doi:10.1038/387094a0 (1997).

31 Ste Marie, L., Luquet, S., Curtis, W. \& Palmiter, R. D. Norepinephrine- and epinephrinedeficient mice gain weight normally on a high-fat diet. Obes Res 13, 1518-1522, doi:10.1038/oby.2005.185 (2005).

32 Karadi, Z. et al. Responses of lateral hypothalamic glucose-sensitive and glucoseinsensitive neurons to chemical stimuli in behaving rhesus monkeys. J Neurophysio/67, 389-400 (1992).

33 Nishino, H., Oomura, Y., Aou, S. \& Lenard, L. Catecholaminergic mechanisms of feeding-related lateral hypothalamic activity in the monkey. Brain Res 405, 56-67 (1987).

$34 \mathrm{Li}, \mathrm{Y}$. \& van den Pol, A. N. Direct and indirect inhibition by catecholamines of hypocretin/orexin neurons. J Neurosci 25, 173-183, doi:10.1523/JNEUROSCI.401504.2005 (2005).

35 Atasoy, D., Aponte, Y., Su, H. H. \& Sternson, S. M. A FLEX switch targets Channelrhodopsin-2 to multiple cell types for imaging and long-range circuit mapping. $J$ Neurosci 28, 7025-7030, doi:10.1523/JNEUROSCI.1954-08.2008 (2008).

36 Schnutgen, F. et al. A directional strategy for monitoring Cre-mediated recombination at the cellular level in the mouse. Nat Biotechnol 21, 562-565, doi:10.1038/nbt811 (2003).

37 Robertson, S. D., Plummer, N. W., de Marchena, J. \& Jensen, P. Developmental origins of central norepinephrine neuron diversity. Nat Neurosci 16, 1016-1023, doi:10.1038/nn.3458 (2013).

38 McCall, J. G. et al. CRH Engagement of the Locus Coeruleus Noradrenergic System Mediates Stress-Induced Anxiety. Neuron 87, 605-620, doi:10.1016/j.neuron.2015.07.002 (2015).

39 Palkovits, M. et al. Noradrenergic innervation of the rat hypothalamus:experimental biochemical and electron microscopic studies. Brain Res 191, 161-171 (1980). 
40 McCall, J. G. et al. Locus coeruleus to basolateral amygdala noradrenergic projections promote anxiety-like behavior. Elife 6, doi:10.7554/eLife.18247 (2017).

41 Bouret, S. \& Richmond, B. J. Sensitivity of locus ceruleus neurons to reward value for goal-directed actions. J Neurosci 35, 4005-4014, doi:10.1523/JNEUROSCI.455314.2015 (2015).

42 Berridge, C. W. \& Waterhouse, B. D. The locus coeruleus-noradrenergic system: modulation of behavioral state and state-dependent cognitive processes. Brain Res Brain Res Rev 42, 33-84 (2003).

43 Bouret, S. \& Sara, S. J. Network reset: a simplified overarching theory of locus coeruleus noradrenaline function. Trends Neurosci 28, 574-582, doi:10.1016/j.tins.2005.09.002 (2005).

44 Tovar, S. et al. K(ATP)-channel-dependent regulation of catecholaminergic neurons controls BAT sympathetic nerve activity and energy homeostasis. Cell Metab 18, 445 455, doi:10.1016/j.cmet.2013.08.006 (2013).

45 Bello, N. T., Yeh, C. Y., Verpeut, J. L. \& Walters, A. L. Binge-like eating attenuates nisoxetine feeding suppression, stress activation, and brain norepinephrine activity. PLoS One 9, e93610, doi:10.1371/journal.pone.0093610 (2014).

46 Redmond, D. E., Jr., Huang, Y. H., Snyder, D. R., Maas, J. W. \& Baulu, J. Hyperphagia and hyperdipsia after locus coeruleus lesions in the stumptailed monkey. Life Sci 20, 1619-1628 (1977).

47 Ahlskog, J. E. \& Hoebel, B. G. Overeating and obesity from damage to a noradrenergic system in the brain. Science 182, 166-169 (1973).

48 Anlezark, G. M., Crow, T. J. \& Greenway, A. P. Impaired learning and decreased cortical norepinephrine after bilateral locus coeruleus lesions. Science 181, 682-684 (1973).

49 Kubiak, P. \& Zagrodzka, J. DSP-4 lesion of locus coeruleus does not affect spontaneous predatory behaviour in cats. Acta Neurobiol Exp (Wars) 53, 525-534 (1993).

50 Leibowitz, S. F. Reciprocal hunger-regulating circuits involving alpha- and betaadrenergic receptors located, respectively, in the ventromedial and lateral hypothalamus. Proc Natl Acad Sci U S A 67, 1063-1070 (1970).

51 Margules, D. L., Lewis, M. J., Dragovich, J. A. \& Margules, A. S. Hypothalamic norepinephrine: circadian rhythms and the control of feeding behavior. Science 178, 640-643 (1972).

52 Margules, D. L. Alpha-adrenergic receptors in hypothalamus for the suppression of feeding behavior by satiety. J Comp Physiol Psychol 73, 1-12 (1970).

53 Capuano, C. A., Leibowitz, S. F. \& Barr, G. A. The pharmaco-ontogeny of the perifornical lateral hypothalamic beta 2-adrenergic and dopaminergic receptor systems mediating epinephrine- and dopamine-induced suppression of feeding in the rat. Brain Res Dev Brain Res 70, 1-7 (1992). 
54 Booth, D. A. Localization of the adrenergic feeding system in the rat diencephalon. Science 158, 515-517 (1967).

55 Brown, J. A., Woodworth, H. L. \& Leinninger, G. M. To ingest or rest? Specialized roles of lateral hypothalamic area neurons in coordinating energy balance. Front Syst Neurosci 9, 9, doi:10.3389/fnsys.2015.00009 (2015).

56 Stuber, G. D. \& Wise, R. A. Lateral hypothalamic circuits for feeding and reward. Nat Neurosci 19, 198-205, doi:10.1038/nn.4220 (2016).

57 Bonnavion, P., Mickelsen, L. E., Fujita, A., de Lecea, L. \& Jackson, A. C. Hubs and spokes of the lateral hypothalamus: cell types, circuits and behaviour. J Physiol 594, 6443-6462, doi:10.1113/JP271946 (2016).

58 Qualls-Creekmore, E. \& Munzberg, H. Modulation of Feeding and Associated Behaviors by Lateral Hypothalamic Circuits. Endocrinology, doi:10.1210/en.2018-00449 (2018).

59 Cassidy, R. M. et al. A lateral hypothalamus to basal forebrain neurocircuit promotes feeding by suppressing responses to anxiogenic environmental cues. Sci Adv 5, eaav1640, doi:10.1126/sciadv.aav1640 (2019).

60 Grivel, J. et al. The wake-promoting hypocretin/orexin neurons change their response to noradrenaline after sleep deprivation. J Neurosci 25, 4127-4130, doi:10.1523/JNEUROSCI.0666-05.2005 (2005).

61 Gao, X. B., Ghosh, P. K. \& van den Pol, A. N. Neurons synthesizing melaninconcentrating hormone identified by selective reporter gene expression after transfection in vitro: transmitter responses. J Neurophysio/ 90, 3978-3985, doi:10.1152/jn.00593.2003 (2003).

62 Arnsten, A. F. \& Li, B. M. Neurobiology of executive functions: catecholamine influences on prefrontal cortical functions. Biol Psychiatry 57, 1377-1384, doi:10.1016/j.biopsych.2004.08.019 (2005). 
a
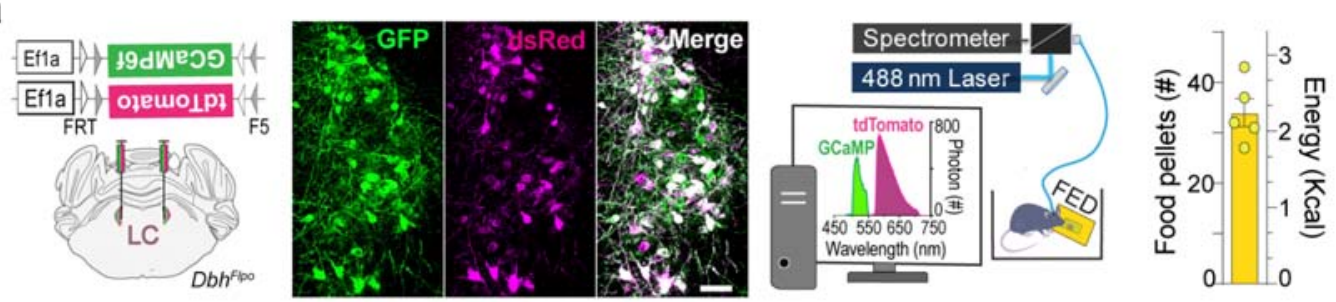

b
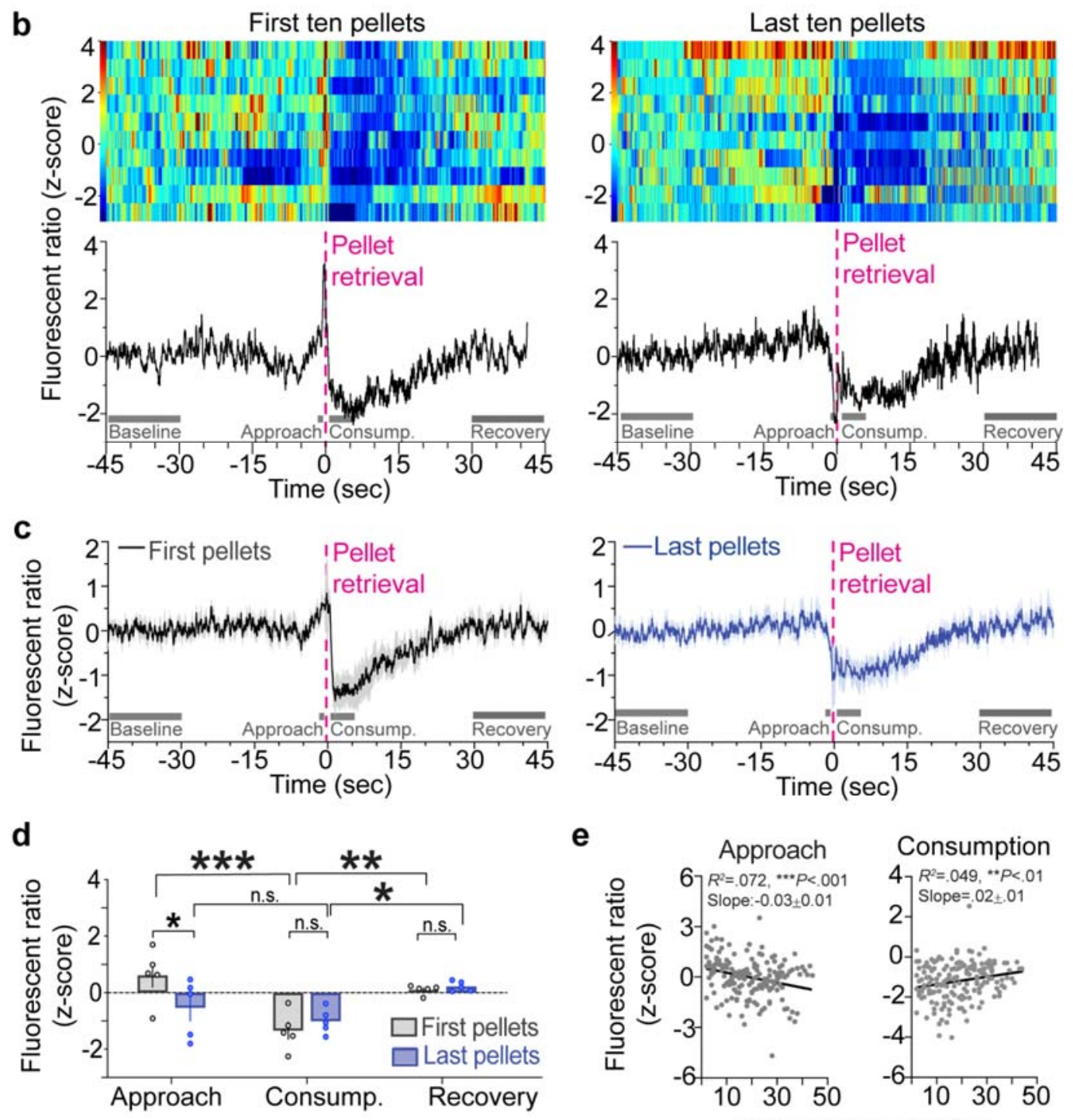

e

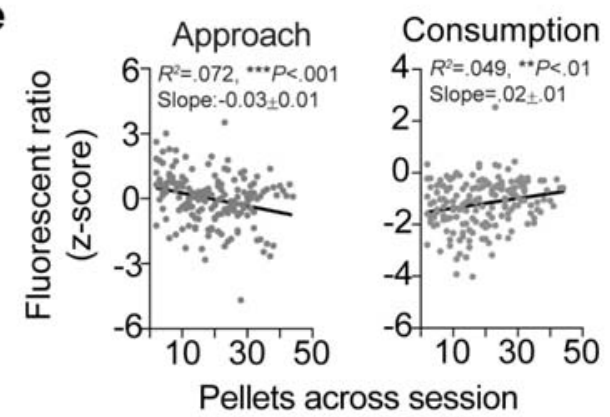

Figure 1. Activity of LC-NE neurons is increased during food approach and suppressed during feeding in a manner influenced by nutritional state. (a) Top. Flp-dependent viral genetic strategy for co-expression of GCaMP6f and tdTomato in LC-NE neurons. Middle. Coronal view of the locus coeruleus from a LC GCaMP/tdT mouse immunostained for GCaMP6f 
(GFP antibody) and tdTomato (dsRed antibody). Schematic of in vivo fiber photometry setup and feeding experimentation device (FED). Right. Average energy intake across the 1-hr session. Data are mean \pm SEM. $n=5$ fasted LC $\mathrm{GCaMP} / \mathrm{ddT}^{\text {mice. }}$ (b) Example response around pellet retrieval of a fasted mouse expressing a fluorescent ratio of GCaMP6f/tdTomato in LC neurons during the first ten pellets (left) and last ten pellets of the session (right). Single trials are represented in the heat map, whereas the average $z$-score fluorescent ratio is represented in the trace below. (c) Average z-score fluorescent ratio aligned-to-pellet retrieval in fasted mice during the first ten pellets (left) and last ten pellets of the session (right). Data are mean \pm SEM. $n=5$ LC $^{\mathrm{GCaMP} / \mathrm{tdT}}$ mice. (d) Average z-score fluorescent ratio during feeding-related behaviors. Two-way repeated measures ANOVA, nutritional state $\mathrm{x}$ behavior interaction: $F_{2,8}=7.018$, $P=0.0174$. Bonferroni post-hoc test ${ }^{* *} P<0.001,{ }^{* *} P<0.01$, and ${ }^{*} P<0.05$. Data are mean \pm SEM. $n=5 \mathrm{LC}^{\mathrm{GCaMP} / \mathrm{dT}}$ mice. (e) Linear regression of the average fluorescent ratio during food approach and consumption in relation to pellet events across the session in LC GCaMP/tdT mice following an overnight fast. Approach $\left(R^{2}=0.072 ; \quad F_{1,168}=13.06, \quad P<0.001\right)$ and Consumption $\left(R^{2}=0.049\right.$; $\left.F_{1,168}=8.05,{ }^{* *} P<0.01\right)$. 
a
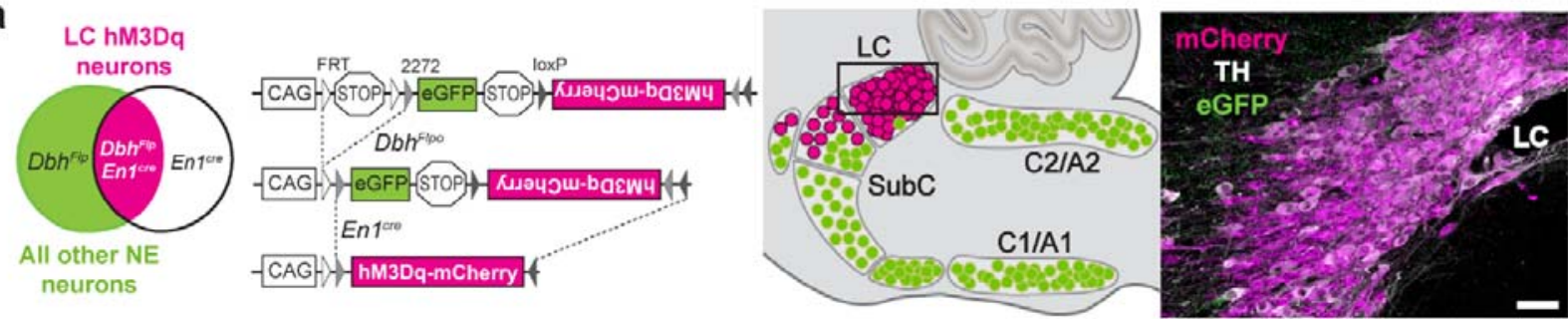

b

- CNO-LChM3Dq

- Veh-LC

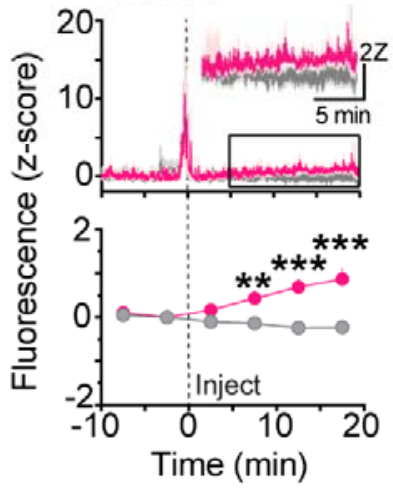

d
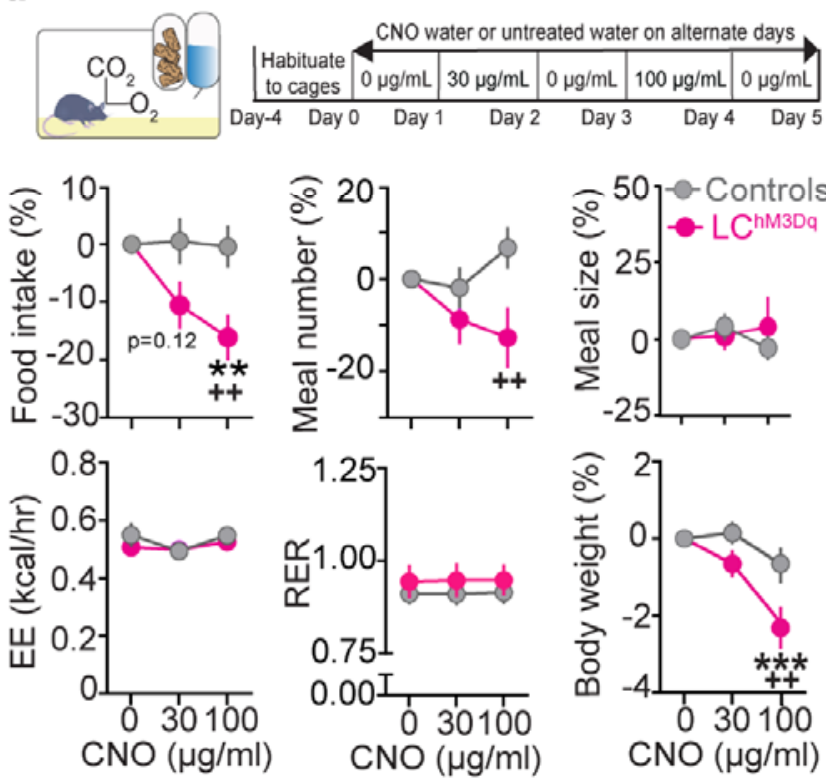
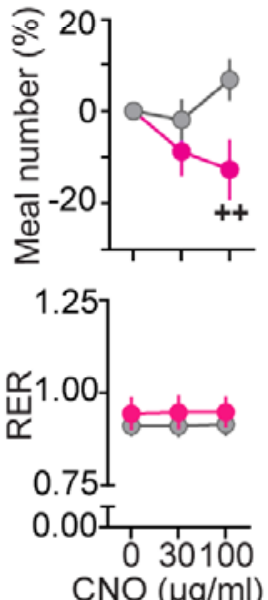

- CNO-LCGFP
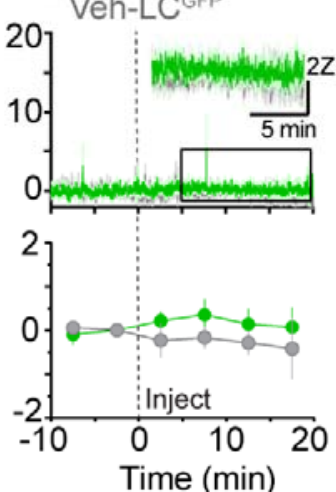

- Veh-LCGPP
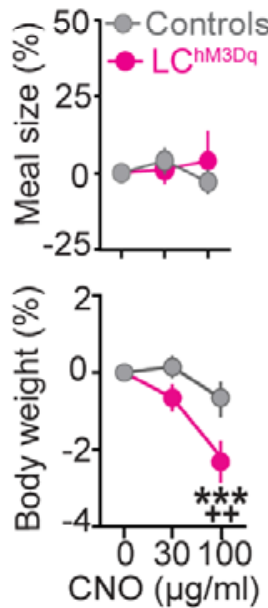

- Yohimbine

- Veh
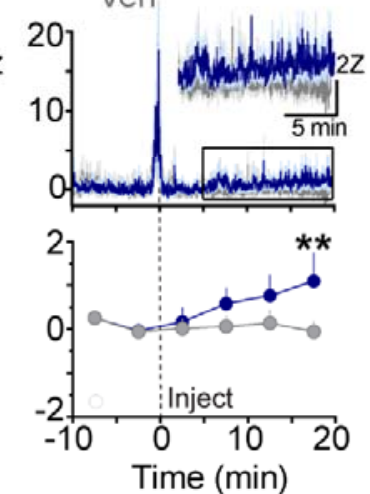

e

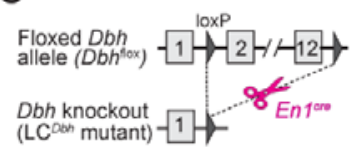

C

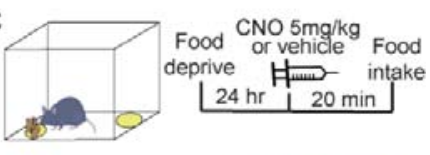

$\square$ Controls $\square$ LC $^{\text {hM3Da }}$

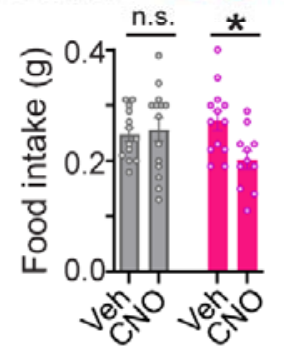

$\mathbf{f}$

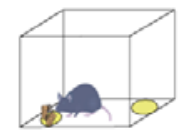

$\mathrm{YOH} 3 \mathrm{mg} / \mathrm{kg}$

Food or vehicle Food deprive \begin{tabular}{|l|l|}
$24 \mathrm{hr}$ & $20 \mathrm{~min}$ \\
\hline
\end{tabular}

$\square$ Vehicle

Yohimbine

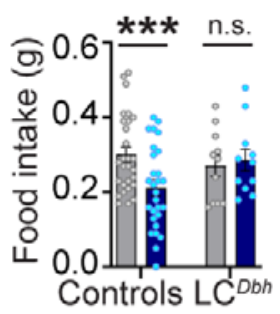

Figure 2. Activation of LC-NE neurons suppresses feeding in a manner dependent on

norepinephrine signaling. (a) Left. Schematic illustration of intersectional genetic strategy.

Recombination of $R C:: F L-h M 3 D q$ allele by $D b h^{F / p o}$ and $E n 1^{\text {cre }}$ results in LC-selective hM3DqmCherry expression. Recombination by $D b h^{F / p o}$ alone leads to eGFP expression. Right. Schematic shows a mouse hindbrain compressed across the sagittal axis. Parasagittal section 
from LC ${ }^{\mathrm{hM} 3 \mathrm{Dq}}$ brain reveals hM3Dq-mCherry expression in LC-NE neurons. Scale, $50 \mu \mathrm{m}$. (b) Top. Average z-score fluorescence aligned to injection time in $\mathrm{LC}^{\mathrm{hM} 3 \mathrm{Da}}$ mice expressing either GCaMP6f or GCaMP6f/tdTomato treated with clozapine n-oxide (CNO; magenta), yohimbine (blue) or vehicle (gray), and in LC GFP mice treated with CNO (green) or vehicle (gray). In all mice, except LC GFP controls, we detected a transient increase in LC-NE activity in response to an experimenter approaching for injection, demonstrating the sensitivity of our recording conditions to detect an established visual threat response characterized previously ${ }^{3,4,14}$. Bottom. Quantification of average z-score fluorescence following CNO (5 mg/kg i.p.) or yohimbine (3 mg/kg i.p.) treatment. Two-way repeated measures ANOVA, drug $x$ time interaction: LC ${ }^{\text {hM3Dq }}\left(F_{5}\right.$, $35=9.157, P=0.0001), \operatorname{LC}^{\mathrm{GFP}}\left(F_{5,20}=0.4994, P=0.7730\right)$, LC-Yohimbine $\left(F_{5,20}=2.592, P=0.0579\right)$. Bonferroni post-hoc, ${ }^{* * *} P<0.01,{ }^{* *} P<0.01$ vs. vehicle, $n=8$ LC ${ }^{\text {hM3Da }}$ mice. $n=5$ LC ${ }^{\text {GFP }}$ mice. $n=5$ mice for yohimbine-LC experiment. Data are mean \pm SEM. (c) Top. Timeline of acute food intake experiments in fasted mice. Bottom. Average food intake in fasted mice. Two-way between subject's ANOVA, drug $\mathrm{x}$ genotype interaction: $F_{1,47}=5.2, P=0.0272$. Bonferroni posthoc test, ${ }^{*} P<0.05 . n=13$ vehicle-treated and $n=14$ CNO-treated littermate controls. $n=13$ vehicle-treated and $n=11$ CNO-treated LC ${ }^{\mathrm{hM} 3 \mathrm{Da}}$ mice. (d) Top. Timeline of CNO water dosing at 30 and $100 \mu \mathrm{g} / \mathrm{mL}$. Note that this method delivered a daily total dose of approximately 4 or 10 $\mathrm{mg} / \mathrm{kg}$ CNO. Bottom. Measures in the automated homecage test. Two-way repeated measures ANOVA, drug x genotype interaction: food intake $\left(F_{2,54}=3.59, P=0.0343\right)$, meal number $\left(F_{2,54}=\right.$ 3.44, $P=0.0393)$, meal size $\left(F_{2,54}=0.864, P=0.4271\right)$, energy expenditure $\left(E E, F_{2,54}=0.22\right.$, $P=0.8036)$, respiratory exchange rate $\left(\mathrm{RER}, F_{2,54}=0.0509, P=0.9504\right)$, and body weight $\left(F_{2,70}=\right.$ 3.65, $P=0.031$ ). Bonferroni posthoc test, ${ }^{* *} P<0.001,{ }^{* *} P<0.01$ vs. vehicle; ${ }^{++} P<0.01$ vs. littermate controls. $n=20$ littermate controls. $n=9$ LC ${ }^{\text {hM3Dq }}$ mice for all measures (expect body weight wherein $\mathrm{n}=17 \mathrm{LC} \mathrm{hM3Da}^{\mathrm{mice}}$ ). Data are mean \pm SEM. (e) Top. Schematic diagram of $D b h$ cKO allele. Recombination by En1 ${ }^{\text {cre }}$ leads to disruption of norepinephrine synthesis in LC-NE neurons. Middle. Coronal brain sections stained for Dbh (riboprobe; magenta) and tyrosine 
hydroxylase (TH antibody; green). Scale, 50- $\mu \mathrm{m}$. Bottom. Two-way repeated subject's ANOVA, time $x$ genotype interaction: $F_{6,114}=0.77, P=0.5925 . n=18-19$ littermate controls, $n=11-13$ LC $^{D b h}$ mutants. Data are \pm SEM. (f) Food intake in fasted mice treated with yohimbine (3 mg/kg, i.p.). Two-way repeated measures ANOVA, drug $\mathrm{x}$ genotype interaction: $F_{1,37}=10.8, P<0.0022$. Bonferroni posthoc test, ${ }^{* * *} P<0.001$ vs vehicle. n.s., non-significant. $n=28$ littermate controls, $n=11$ LC $^{D b h}$ mutants. Data are \pm SEM. 

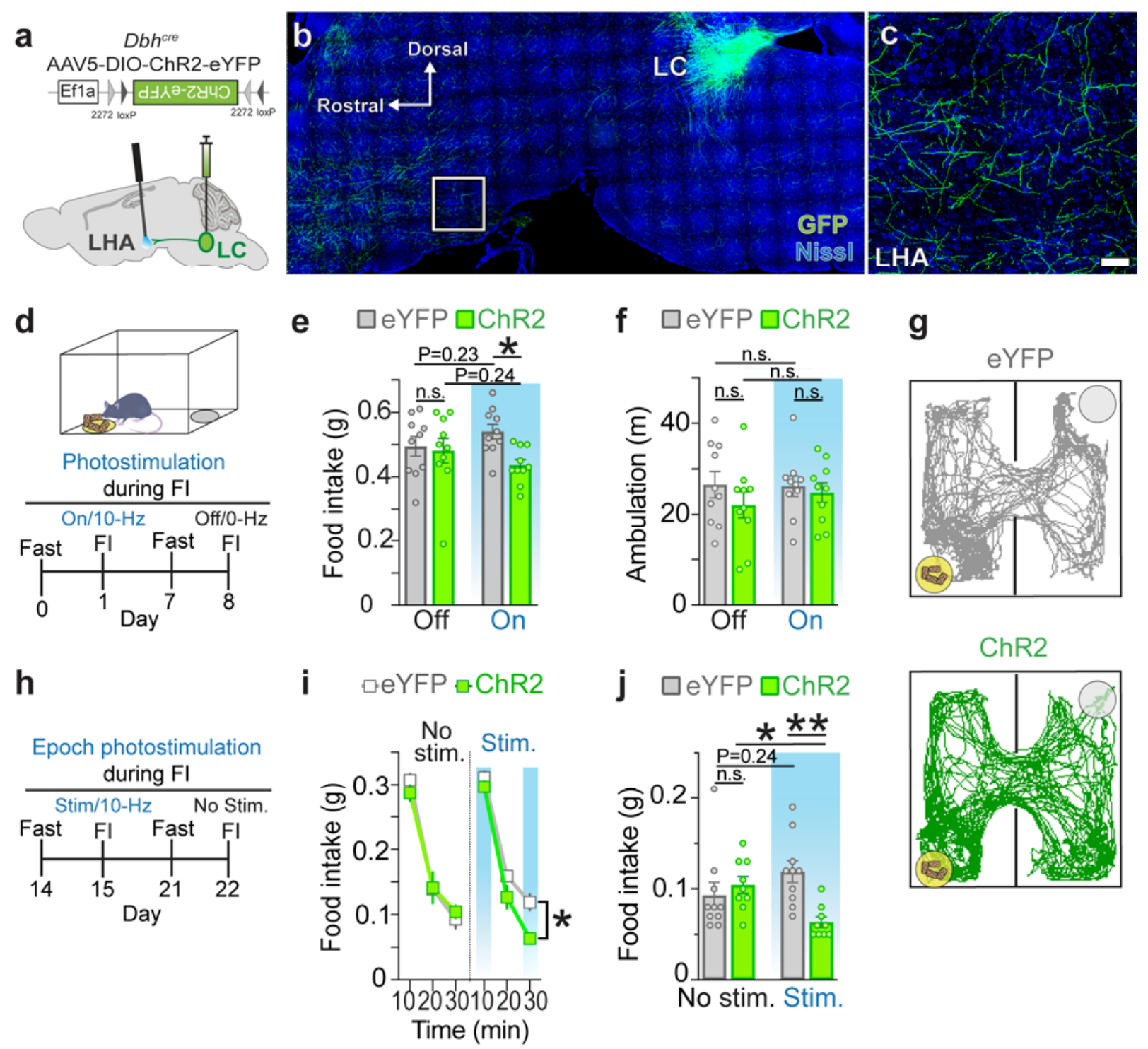

Figure 3. Stimulation of LC-LHA noradrenergic circuit suppresses feeding. (a) Schematic illustration of sagittal mouse brain shows location of cre-dependent AAV used to drive ChR2eYFP expression and location of fiberoptic probes. (b-c) Parasagittal brain section from a $D b h^{\text {cre }}$; eYFP ${ }^{\mathrm{LC} \rightarrow \mathrm{LHA}}$ mouse shows restricted eYFP expression in LC-NE neurons. High magnification image shows eYFP-expressing LC-NE axonal projections in the LHA. Scale is 400- $\mu \mathrm{m}$ (brain) and 150- $\mu \mathrm{m}$ (LHA). (d) Timeline of photostimulation (10-Hz, 10-ms pulses) during food intake (FI) test. (e-f) Feeding-related behaviors in fasted mice. Two-way repeated measure ANOVA, Stimulation $x$ Virus interaction: food intake $\left(e, F_{1,18}=5.44, P=0.0314\right)$ and 
ambulation (f, $\left.F_{1,18}=0.655, P=0.4290\right)$, Bonferroni posthoc test, ${ }^{*} P<0.05 . n=10$ eYFP ${ }^{\text {LC-LHA }}$ mice, $\mathrm{n}=10 \mathrm{ChR} 2^{\mathrm{LC}-\mathrm{LHA}}$ mice. Data are mean \pm SEM. (g) Representative traces shows ambulation in the feeding task during photostimulation. Yellow circle indicates the location of the food cup and gray circle indicates location of the empty cup. (h) Timeline of epoch photostimulation (10-Hz, 10-ms pulses) during food intake. (i-j) Food intake in fasted mice during entire epoch experiment (i) and last epoch bin (j). Three-way repeated measure ANOVA, Stimulation x Virus x Time interaction: (i, $\left.F_{2,34}=3.646, P=0.037\right)$ and Time main effect $\left(\mathbf{i}, F_{2,34}=295.996, P<0.001\right)$. Two-way repeated measure ANOVA, Stimulation $x$ Virus interaction $\left(\mathrm{j}, F_{1,17}=8.57, P=0.0094\right)$. Bonferroni posthoc test, ${ }^{* *} P<0.01,{ }^{*} P<0.05$ ChR2 vs. eYFP during second photostimulation epoch. $n=10$ eYFP ${ }^{\mathrm{LC}-\mathrm{LHA}}$ mice, $\mathrm{n}=9 \mathrm{ChR} 2^{\mathrm{LC}-\mathrm{LHA}}$ mice. Data are mean \pm SEM. n.s., non-significant. 